\title{
Good Prognosis of Localized Osteosarcoma in Young Patients Treated with Limb-Salvage Surgery and Chemotherapy
}

\begin{tabular}{|c|c|}
\hline Journal: & Pediatric Blood \& Cancer \\
\hline Manuscript ID: & PBC-10-0637.R3 \\
\hline Wiley - Manuscript type: & Research Article \\
\hline $\begin{array}{r}\text { Date Submitted by the } \\
\text { Author: }\end{array}$ & $\mathrm{n} / \mathrm{a}$ \\
\hline Complete List of Authors: & $\begin{array}{l}\text { Hegyi, Marta; Semmelweis University, 2nd Department of Pediatrics } \\
\text { Semsei, Agnes; Semmelweis University, Department of Genetics, } \\
\text { Cell- and Immunobiology } \\
\text { Jakab, Zsuzsanna; Semmelweis University, 2nd Department of } \\
\text { Pediatrics } \\
\text { Antal, Imre; Semmelweis University, Department of Orthopedics } \\
\text { Kiss, János; Semmelweis University, Department of Orthopedics } \\
\text { Szendrői, Miklós; Semmelweis University, Department of } \\
\text { Orthopedics } \\
\text { Csóka, Monika; Semmelweis University, 2nd Department of } \\
\text { Pediatrics } \\
\text { Kovács, Gábor; Semmelweis University, 2nd Department of } \\
\text { Pediatrics }\end{array}$ \\
\hline Keywords: & $\begin{array}{l}\text { Osteosarcoma, Pediatric oncology, Outcomes research, } \\
\text { Chemotherapy, Prognosis }\end{array}$ \\
\hline
\end{tabular}

\section{SCHOLARONE Manuscripts}




\section{Good Prognosis of Localized Osteosarcoma in Young Patients Treated with Limb-Salvage Surgery and Chemotherapy}

\footnotetext{
Marta Hegyi MD ${ }^{1}$, Agnes F. Semsei MSc ${ }^{2}$, Zsuzsanna Jakab MD, PhD ${ }^{1}$, Imre Antal MD, PhD

${ }^{3}$, Janos Kiss $\mathrm{MD}, \mathrm{PhD}^{3}$, Miklos Szendroi MD, $\mathrm{PhD}^{3}$, Monika Csoka $\mathrm{MD}, \mathrm{PhD}^{1}$, Gabor Kovacs $\mathrm{MD}, \mathrm{PhD}^{1}$
}

${ }^{1}$ 2nd Department of Pediatrics, Semmelweis University, Budapest, Hungary

${ }^{2}$ Department of Genetics, Cell- and Immunobiology, Semmelweis University, Budapest, Hungary

${ }^{3}$ Department of Orthopedics, Semmelweis University, Budapest, Hungary

Correspondence to:

Marta Hegyi, MD, PhD student of the 2nd Department of Pediatrics, Semmelweis University, Budapest, Hungary, Tüzoltó street 7.-9., 1094 Budapest, Tel.: 0036-20-5977193, Email: marta.hegyi@gmail.com

Abstract word count: 226

Text word count: 2903

Brief running title: Osteosarcoma in Hungarian children

Key words: osteosarcoma, pediatric oncology, prognosis, outcome research, chemotherapy Tables: 3

Figures: 5 


\section{Abstract}

Background. The objective of this report was to estimate long-term outcome and prognostic factors in children and adolescents with osteosarcoma. A large group of osteosarcoma patients were analyzed at our national oncology center.

Procedure. To evaluate the efficacy of surgery and multiagent chemotherapy for treating osteosarcoma, we reviewed 122 cases (65 male, 57 female, mean age $13.8 \pm 3.6$ years) treated at the Second Department of Pediatrics in Budapest between 1988 and 2006. Demographic parameters, tumor-related and treatment-related variables, response, overall survival (OS) and event-free survival (EFS) were analyzed.

Results. The 5-year OS was $68 \%$ and 5-year EFS was $62 \%$. OS of patients without metastasis was $79 \%$, while OS with early metastasis was $17 \%$. Survival of patients with amputation $(n=30)$ was not significantly different from patients with limb-salvage surgery $(n=82)$, but all patients without radical surgery died. Gender and histological classification had no prognostic significance. Patients with localized tumors in extremities had increased survival compared to patients with axial skeleton tumors $(\mathrm{p}=0.013)$. Poor histological response to neoadjuvant chemotherapy (rate of survivor tumor cells $>10 \%$ ) was associated with decreased survival $(\mathrm{p}=$ 0.018). Patients under 14 years had better EFS than patients over 14 years $(p=0.008)$.

Conclusions. Our results demonstrate that younger patients with localized osteosarcoma of the extremities who receive limb-salvage surgery and chemotherapy have an excellent survival. 


\section{Introduction}

Osteosarcoma is the most common primary malignant bone tumor in children and adolescents, yet its absolute incidence among malignant tumors is low. The incidence of newly diagnosed cases peaks in the second decade of life (1). As osteosarcoma may produce various kinds of extra cellular matrix and have different degrees of differentiation, huge variability exists in histological patterns. Conventional osteosarcoma can be divided into osteoblastic, chondroblastic, and fibroblastic subtypes depending on the predominant type of extracellular matrix (2). The etiology of osteosarcoma in young patients is unexplained. Osteosarcoma could potentially derive from any cell on the differentiation pathway between a mesenchymal stem cell and a mature osteoblast (3).

The goal of bone sarcoma therapy is curing the patient of both the primary tumor and all (micro-) metastatic deposits while maintaining maximal extremity function and minimalizing treatmentspecific late side effects. Bone sarcoma therapy involves close multidisciplinary coordination of oncologists, orthopedic surgeons, musculoskeletal pathologists and radiologists. It usually consists of induction neoadjuvant chemotherapy followed by local therapy of the primary tumor and any primary metastases, and adjuvant chemotherapy. Local treatment for osteosarcoma should be surgery whenever possible (4). After the introduction of adjuvant chemotherapy in the 1970s, survival has increased from $10-20 \%$ to $50-80 \%$. Doxorubicin, cisplatin, ifosfamide and methotrexate are currently the most effective agents for patients with osteosarcoma (5). Advances in imaging, new materials, and prostheses have assisted surgeons in making more accurate preoperative plans and giving them a broader range of operative alternatives. 
Chemotherapy response; presence of metastatic disease; tumor size and site, with axial locations worse then location of extremities, are reported to be independent prognostic factors of osteosarcoma (6). At the time of diagnosis, $20 \%$ of patients already present with distant metastases, with lung the most frequent metastatic site (5). The prognosis of patients with recurrent disease or metastases at diagnosis is poor. Resection of pulmonary metastases is a standard treatment for lung relapse of a tumor. Survival of patients who have surgery is $36 \%$ after 5 years, and $26 \%$ after 10 years (7). Unresectable tumors increase the risk of local recurrence and result in poor prognosis. (4)

In this retrospective analysis, we collected data on children with osteosarcoma diagnosed between 1988 and 2006 in the Second Department of Pediatrics in Budapest. This disease is rare, so the aim of our study was to examine prognostic significance of factors based on a large cohort of patients homogeneous for ethnic group and treatment. 


\section{Methods}

Between 1988 and 2006, 172 pediatric patients (data collected by the National Pediatric Tumor Registry of Hungary) were diagnosed with osteosarcoma in Hungary. Osteosarcoma patients could be treated at three pediatric oncology centers in the country. The largest is the Second Department of Pediatrics in Budapest, where $70 \%$ of the subjects were treated (122 patients). We retrospectively collected clinical data on these patients (65 males and 57 females; mean age at diagnosis: $13.8 \pm 3.6$ standard deviation [SD], range: 5.5-17.6 years).

The patients were treated according to the Cooperative Osteosarcoma Study Group (COSS) 86 (8) and later COSS 96 protocols (9) including neoadjuvant and adjuvant chemotherapy, and radical surgery (Figure 1). The differences between the two protocols were that the COSS 96 protocol had a shorter low-risk arm and introduced a new branch with increased methotrexate. In Hungary, we used only the standard arms. We did not randomize the study, so the two protocols had no relevant differences in drugs, dosage, or schedule.

Neoadjuvant chemotherapy included one course of doxorubicin $\left(90 \mathrm{mg} / \mathrm{m}^{2}\right)$, two courses of highdose methotrexate $\left(12 \mathrm{~g} / \mathrm{m}^{2}\right)$, and two courses of ifosfamide $(2 \times 3 \mathrm{~g} / \mathrm{m} 2)$ plus cisplatin $(120$ $\mathrm{mg} / \mathrm{m}^{2}$ ). The surgical decision about surgery type was based on tumor volume at the time of diagnosis, and on X-ray and Magnetic Resonance imaging (MRI) examinations before and after neoadjuvant chemotherapy. In addition, orthopedic surgeons estimated whether the resection margin was large enough, and if the salvaged limb would be functional. After radical operation of the tumor (amputation or limb-sparing surgery) patients were classified into risk groups and an additional 6-8 months of chemotherapy was administered. Classification into a risk group was based on the initial volume of the tumor and chemotherapy response of the tumor cells (Table III). Histological response to chemotherapy was characterized by the degree of necrosis detected 
by histological examination of multiple tumor sections after neoadjuvant chemotherapy and surgery. According to the COSS protocols, patients were defined as good responders if less than $10 \%$ of the tumor cells were alive after neoadjuvant therapy, or poor responders if the extent of necrosis was less than 90\%. For adjuvant chemotherapy, the new drugs carboplatin (4 x 150 $\mathrm{mg} / \mathrm{m}^{2}$ ) and etoposid (4 x $150 \mathrm{mg} / \mathrm{m}^{2}$ ) were introduced in the high-risk arm. The other arms of the protocol used the same chemotherapeutic agents as for neoadjuvant therapy.

We examined 5-year overall survival (OS) and 5-year event-free survival (EFS). A patient is considered to be cured after a 5-year event-free period. Patients were divided into subgroups by metastasis (none; early, defined as at diagnosis; or late); type of surgery (biopsy, amputation, limb salvage), chemotherapy protocol (COSS 86 or 96), tumor site (axial skeleton, upper or lower extremity), gender (male or female), histological subtypes (osteoblastic, chondroblastic, fibroblastic, or other), histological response to pre-operative chemotherapy (poor or good response); and age at diagnosis (over or under 14 years).

We used StatSoft's STATISTICA v8.0 program for statistical analyses. Survival data were analyzed by the Kaplan-Meier method. For finding relationships between survival and these different factors, actuarial analyses with log-rank tests were used. 


\section{Results}

For the entire cohort, the 5-year OS was 68\% and the EFS 62\% (Figure 2). Results are in Table III. Of the 122 children, 37 had metastasis, local relapse or both (Figure 3). Of 37 cases, 15 had early metastasis at the time of diagnosis, with an OS of $16.6 \%$. In 11 cases, metastases were in the lungs, in 3 cases, in the bone, and in 1 case in the kidney. Only three patients with early metastases survived. In our patient population, 13 children had late metastases with an OS of 41.6\%. All had pulmonary metastases, and four also had metastases in the regional lymphatic glands. Eight had both local relapse and metastasis. Late pulmonary metastases were treated with thoracotomy and postoperative chemotherapy in 13 children, with 5 surviving. The OS of nonmetastatic patients was $79 \%$ (Figure 4A). Dependence of OS on the time of metastasis appearance was significant $(\mathrm{p}=0.00002)$.

Of 23 patients with local relapses, 18 died (Figure 3). All patients with local recurrence relapsed despite primary tumor surgery. Limb salvage surgery was performed on 16 patients before relapse. Six patients had early metastases and local relapse together, and five of these died. Eight patients had late metastases and local relapse together, and six died. Isolated local recurrence was found in 9 patients, with 2 long-term. The median time to develop local recurrence was 16 months from the date of diagnosis (range: 0.4-118.3 months).

We also analyzed by type of surgery (Figure 4B). All patients who did not undergo radical surgery died. Although 30 patients underwent an amputation, in the last 10 years more than $85 \%$ of patients have undergone limb-salvage surgery, with most of them receiving tumor endoprothesis. EFS was $57 \%$ for amputation cases and $68 \%$ for limb-saving surgery cases. The survival rate of patients undergoing amputation was somewhat lower than those receiving limbsalvage surgeries, because orthopedists chose amputation for patients with a larger tumor volume 
or for whom neoadjuvant chemotherapy had been ineffective. These patients have a poor prognosis. No signficant difference in survival was observed between the two types of surgical procedure $(\mathrm{p}=0.3)$, although patients with biopsy only had significantly worse outcomes $(p=0.00005)$.

There was no difference in survival between patients diagnosed before or after $1995(\mathrm{p}=$ 0.6) (Figure 5A). We chose 1995 as the cut-off because COSS 86 was used from 1988-1995, and COSS 96 was used from 1996-2006. In the group treated according to COSS 86, EFS was 57\%; it was $64 \%$ for the group treated with COSS 96.

We analyzed the relationship between EFS and tumor site and found no significant difference in survival if the primary tumor was in the upper or lower extremity $(p=0.7)$. In both cases, EFS was around $60 \%$ (upper extremity $56.3 \%$ vs. lower extremity $65 \%$ ), while tumors of the axial skeleton showed a much worse survival rate of $22.2 \%(p=0.013)$ (Figure 5D).

Boys and girls showed the same survival rates $(\mathrm{p}=0.5)$ (Figure 5B), with an EFS of $65 \%$ for girls and $57 \%$ for boys. We observed no differences in survival rates between osteosarcoma histological subgroups $(\mathrm{p}=0.9)$ (Figure 5C). A significant difference was detected in survival rate between poor and good responders $(\mathrm{p}=0.018)$. The EFS was $77 \%$ for good responders and $50 \%$ for poor responders (Figure 4C). Significantly better results were achieved with patients younger than 14 years than with patients over 14 years $(\mathrm{p}=0.008)$. While the EFS of the patients over 14 years was only $48.4 \%$, the EFS of patients under 14 years was $74.1 \%$ (Figure 4D). We were unable to collect tumor volume data because $60 \%$ of these data were missing or incorrect because poor documentation. 


\section{Discussion}

Osteosarcoma in childhood was curable by the COSS 86 and COSS 96 protocols, with $68 \%$ of our patients achieving long-term survivor status. Previous COSS protocols had poorer survival results. For example, the COSS 77 protocol had an OS of only 47\% (10).

Our results correspond with the international data. The combined use of surgery and multidrug chemotherapy results in 5-year EFS rates of approximately 50-70\% in patients with localized extremity osteosarcoma (4). Ferrari et al. (11) found that patients aged less than 40 years with nonmetastatic osteosarcoma of the extremity had an expected 5-year survival rate of $70 \%$ with a chemotherapy regimen based on methotrexate, cisplatin, doxorubicin, and ifosfamide. Further improvement cannot be achieved by dose intensification of treatment, and new strategies are required. Prolonged follow-up is mandatory due to the risk of late side effects, second tumors and late relapse of osteosarcoma (11). Jaffe established that disease-free survival increased from $<20 \%$ to $55-75 \%$ after the introduction of effective chemotherapy. Furthermore, limb salvage expanded to $80 \%$ of patients (12). An international collaboration between Western Europe, Turkey, Croatia, and the USA examined prognostic factors and outcomes for osteosarcoma and found that the 5-year mortality risk of osteosarcoma was $48 \%$. Most of the 2680 patients (78\%) had combined surgery and chemotherapy, $14 \%$ had surgery alone, $3 \%$ had chemotherapy alone, and $5 \%$ had other combinations or treatments (13). We found that the most important prognostic factors of osteosarcoma are radical resecability of the tumor, extent of disease at diagnosis, initial tumor volume, and initial response of tumor cells to neoadjuvant chemotherapy.

Reconstructive techniques have improved substantially over the past decades, particularly for young patients who have not yet reached skeletal maturity. Studies of osteosarcoma have 
consistently shown an increased risk of local recurrence in patients undergoing limb-salvage surgery, but no increase in mortality. Local recurrence in osteosarcoma is directly associated with the margins of excision and with the responsiveness of the primary tumor to chemotherapy; in patients with a close margin of excision and less than $90 \%$ necrosis of the tumor after neoadjuvant chemotherapy, local recurrence can be as high as $30 \%$ (14). Rozeman et al. found the incidence of local relapses (5-10\%) was higher after limb-salvage surgery than after amputation (0.5-2\%) (5). In our data, the rate of local relapses after limb-salvage surgery was slightly higher (14\%). Approximately $90-95 \%$ of local recurrences appear after limb-salvage surgery, especially when surgical incision is not sufficiently radical. However, amputation to achieve cure has not led to improved survival compared with limb-salvage techniques. The challenge for the surgeon is to reconstruct the limb to allow the child to grow as normally as possible (15).

At least $30-40 \%$ of patients with osteosarcoma develop metastases, of which over $80 \%$ include the lungs and approximately 15\% distant bones. Our results show that the number of lesions at diagnosis has prognostic significance in osteosarcoma. Either presence of metastases or local recurrence triples the mortality risk. The 5-year risk of metastasis was $45 \%$ in Pakkos et al. (13). By multivariate analysis, the presence of metastases increased the risk for a new metastasis fivefold, but no other clinical or demographic characteristics were independently associated with metastasis risk (13). In patients with osteosarcoma of the extremity with lung metastases, a combination of aggressive chemotherapy with simultaneous resection of primary and metastatic tumors can be a therapeutic approach (16). In this study, the mean survival rate of patients with lung metastasis is $10-30 \%$. The length of the relapse-free interval is also an important prognostic factor. In cases of early metastasis, almost all patients died while the OS rate of late metastatic patients was quite good in our study (42\%). 
Initial tumor size is an important prognostic factor in osteosarcoma. When involvement of neighboring tissues is high, radical surgery is more difficult, and the chance of distant metastasis increases, resulting in a lower survival rate in cases of larger tumors. Bacci et al. found that tumor volume greater than $200 \mathrm{~mL}$ was significantly correlated with increased risk of recurrence (17). In a review of St. Jude Children's Research Hospital studies, metastatic disease was associated with tumor diameters over $8 \mathrm{~cm}(\mathrm{p}=0.002)(18)$. For technical reasons, we were not able to analyze the effect of initial tumor volume on prognosis.

Histological tumor response to neoadjuvant chemotherapy after surgery correlates with risk of recurrence. Good responders have less than $10 \%$ viable tumor cells. At our center, their 5-year OS was $84 \%$ and their 5-year EFS was $77.3 \%$, while OS for poor responders was only $55.3 \%$, and EFS was only 50\%. Several international data support the importance of tumor response for survival. Pakkos et al. (13) observed that tumor necrosis was a strong predictor for all major clinical outcomes (13). Another trial of 497 patients showed that intensification of chemotherapy improves histological response without concomitant improvement in progression-free survival or OS (19).

Our results showed that tumors of the axial skeleton had a much worse prognosis than the tumors of the extremities because of the difficulties of radical surgery in this region. Without radical operation, the prognosis for osteosarcoma is very poor. Surgery plays a key role in long-term survival, as only $10 \%$ of patients with osteosarcoma are cured by chemotherapy alone (20).

From the literature, the importance of age at diagnosis as a prognostic factor in osteosarcoma patients is unclear. A better prognosis for younger patients was reported by Bielack et al., who found that patient age at diagnosis (actuarial 10-year survival of patients older than 40 years $=41.6 \%$; patients younger than 40 years $E F S=60.2 \% ; p=0.012$ ) had significant influence on outcome (21). A study by Harting et al. (22) consisted of 438 patients of all ages diagnosed 
with osteosarcoma between 1980 and 2000 who underwent the majority of their treatment at MD Anderson Cancer Center. By univariate analyses, an age of 40 or over was found to be a poor prognostic factor, however age was not identified as a significant prognostic variable by multivariate analysis (22). A retrospective analysis of Szendroi et al. showed that age over 30 years had indirect negative influence on the final outcome through higher intolerability to drugs and lower cooperation from the patient (23). Bacci et al. found that patients 14 years old or younger had an independently worse prognosis than older patients (17). In contrast, in our study, significantly better results were achieved in patients younger than 14 years than in patients over 14 years $(\mathrm{p}=0.008)$. Pakos et al. found that, for 2680 patients with osteosarcoma, survival was worse with older age (7\% relative risk increase per decade) by multivariate analyses (13).

Patients with suspected or confirmed osteosarcoma should be evaluated and treated at a comprehensive cancer center within a multidisciplinary sarcoma program that includes pediatric, medical and radiation oncologists; orthopedic and surgical oncologists; musculoskeletal pathologists; and radiologists. Successful treatment involves adequate diagnosis, preoperative and adjuvant multiagent chemotherapy, and aggressive surgery with an emphasis toward limb preservation. Patients treated for osteosarcoma should be followed closely both for relapse of disease and for development of late effects from chemotherapy (24).

Osteosarcoma is a radioresistant lesion (24). Radiotherapy is used for palliative therapy in patients with radically inoperable tumors. Radiation therapy may represent an alternative to definite surgery in selected patients, in particular those with good response to chemotherapy, for whom surgery is either not feasible or refused (25). We used radiotherapy for palliative treatment in only two cases.

Based on this Hungarian population, we conclude that $68 \%$ of patients are curable through intensive chemotherapy and surgery. Limb-salvage surgery is not associated with a significantly 
increased risk of adverse events, but gives much better quality of life. Patients with metastatic disease or local relapse have a very poor prognosis. Nonmetastatic cases, cases of tumors of the extremities, and patients under 14 years have a survival of $80 \%$. To further improve survival, a continued emphasis should be placed on preclinical basic science and translational research aimed at furthering our understanding of osteosarcoma with the intention of providing patients with new, molecularly targeted therapies.

\section{Acknowledgements}

We are grateful to all the nurses, doctors and patients who took part in the study.

Conflict of interest: There are no competing financial interests in relation to the work described 


\section{References}

1. Messerschmitt PJ, Garcia RM, Abdul-Karim FW, et al. Osteosarcoma. J Am Acad Orthop Surg 2009:17:515-527.

2. Klein MJSiegal GP. Osteosarcoma: Anatomic and histologic variants. Am J Clin Pathol 2006:125:555-581.

3. Li N, Yang R, Zhang W, et al. Genetically transforming human mesenchymal stem cells to sarcomas: Changes in cellular phenotype and multilineage differentiation potential. Cancer 2009:115:4795-4806.

4. Bielack SS, Carrle D, Hardes J, et al. Bone tumors in adolescents and young adults. Curr Treat Options Oncol 2008:9:67-80.

5. Rozeman LB, Cleton-Jansen AMHogendoorn PC. Pathology of primary malignant bone and cartilage tumours. Int Orthop 2006:30:437-444.

6. Bramer JA, van Linge JH, Grimer RJ, et al. Prognostic factors in localized extremity osteosarcoma: A systematic review. Eur J Surg Oncol 2009:35:1030-1036.

7. Briccoli A, Rocca M, Salone M, et al. Resection of recurrent pulmonary metastases in patients with osteosarcoma. Cancer 2005:104:1721-1725. 
8. Bieling P, Bielack S, Delling G, et al. [neoadjuvant chemotherapy of osteosarcoma. Preliminary results of the cooperative coss-86 osteosarcoma study]. Klin Padiatr 1991:203:220-230.

9. Bielack S, Jurgens H, Jundt G, et al. Osteosarcoma: The coss experience. Cancer Treat Res 2009:152:289-308.

10. Bielack S, Kempf-Bielack B, Schwenzer D, et al. [neoadjuvant therapy for localized osteosarcoma of extremities. Results from the cooperative osteosarcoma study group coss of 925 patients]. Klin Padiatr 1999:211:260-270.

11. Ferrari S, Palmerini E. Adjuvant and neoadjuvant combination chemotherapy for osteogenic sarcoma. Curr Opin Oncol 2007:19:341-346.

12. Jaffe N. Osteosarcoma: Review of the past, impact on the future. The american experience. Cancer Treat Res 2009:152:239-262.

13. Pakos EE, Nearchou AD, Grimer RJ, et al. Prognostic factors and outcomes for osteosarcoma: An international collaboration. Eur J Cancer 2009:45:2367-2375.

14. Picci P, Sangiorgi L, Bahamonde L, et al. Risk factors for local recurrences after limbsalvage surgery for high-grade osteosarcoma of the extremities. Ann Oncol 1997:8:899-903. 
15. Grimer RJ. Surgical options for children with osteosarcoma. Lancet Oncol 2005:6:85-

92.

16. Bacci G, Briccoli A, Ferrari S, et al. Neoadjuvant chemotherapy for osteosarcoma of the extremities with synchronous lung metastases: Treatment with cisplatin, adriamycin and high dose of methotrexate and ifosfamide. Oncol Rep 2000:7:339-346.

17. Bacci G, Longhi A, Versari M, et al. Prognostic factors for osteosarcoma of the extremity treated with neoadjuvant chemotherapy: 15-year experience in 789 patients treated at a single institution. Cancer 2006:106:1154-1161.

18. Rodriguez-Galindo C, Liu T, Krasin MJ, et al. Analysis of prognostic factors in ewing sarcoma family of tumors: Review of st. Jude children's research hospital studies. Cancer 2007:110:375-384.

19. Lewis IJ, Nooij MA, Whelan J, et al. Improvement in histologic response but not Survival in osteosarcoma patients treated with intensified chemotherapy: A randomized phase iii trial of the european osteosarcoma intergroup. J Natl Cancer Inst 2007:99:112128.

20. Jaffe N, Carrasco H, Raymond K, et al. Can cure in patients with osteosarcoma be achieved exclusively with chemotherapy and abrogation of surgery? Cancer 2002:95:22022210. 
21. Bielack SS, Kempf-Bielack B, Delling G, et al. Prognostic factors in high-grade osteosarcoma of the extremities or trunk: An analysis of 1,702 patients treated on neoadjuvant cooperative osteosarcoma study group protocols. J Clin Oncol 2002:20:776790.

22. Harting MT, Lally KP, Andrassy RJ, et al. Age as a prognostic factor for patients with osteosarcoma: An analysis of 438 patients. J Cancer Res Clin Oncol 2010:136:561-570.

23. Szendroi M, Papai Z, Koos R, et al. Limb-saving surgery, survival, and prognostic factors for osteosarcoma: The hungarian experience. J Surg Oncol 2000:73:87-94.

24. Federman N, Bernthal N, Eilber FC, et al. The multidisciplinary management of osteosarcoma. Curr Treat Options Oncol 2009:10:82-93.

25. Hundsdoerfer P, Albrecht M, Ruhl U, et al. Long-term outcome after polychemotherapy and intensive local radiation therapy of high-grade osteosarcoma. Eur J Cancer 2009:45:2447-2451. 
Table I. Definition of the risk groups

\begin{tabular}{ll}
\hline Risk group & Definition \\
\hline Low risk group & tumor volume $\leq 70 \mathrm{ml}$ without reference to histological regression \\
& or tumor volume: $71-150 \mathrm{ml}$ and the histological regression I/II \\
Standard risk group & tumor volume: $71-150$ and the histological regression III-IV \\
& or tumor volume $\geq 150 \mathrm{ml}$ and the histological regression I-IV \\
High risk group & tumor volume $\geq 150 \mathrm{ml}$ and the histological regression V/VI
\end{tabular}


Table II. Grade of histological regression of osteosarcoma after neoadjuvant chemotherapy

Grade Definition

I. no survivor tumor cells

II. sporadically one-one tumor cell or one survivor tumor cell island which is smaller than 0.20 inch $(0.5 \mathrm{~cm})$

III. rate of the survivor tumor cells less than $10 \%$

IV. rate of the survivor tumor cells $10-50 \%$

V. rate of the survivor tumor cells more than $50 \%$

VI. ineffective chemotherapy 
Table III. Overview of prognostic factors analyzed in our study

\begin{tabular}{|c|c|c|c|c|}
\hline Subgroups & $\begin{array}{l}\text { Overall } \\
\text { survival (\%) }\end{array}$ & $\begin{array}{l}\mathrm{p} \text { value } \\
\text { (OS) }\end{array}$ & $\begin{array}{l}\text { Event-free } \\
\text { survival (\%) }\end{array}$ & $\begin{array}{l}\mathrm{p} \text { value } \\
\text { (EFS) }\end{array}$ \\
\hline Whole cohort & 68.0 & & 62 & \\
\hline \multicolumn{5}{|l|}{ Metastasis } \\
\hline No metastasis & 79 & & & \\
\hline Early metastasis & 16.6 & 0.00002 & & \\
\hline Late metastasis & 41.6 & & & \\
\hline \multicolumn{5}{|l|}{ Type of surgery } \\
\hline Biopsy & 0 & & 0 & \\
\hline Amputation & 63.3 & $<0.00005$ & 56.6 & 0.00005 \\
\hline Limb salvage & 76.9 & & 68.3 & \\
\hline \multicolumn{5}{|l|}{ Chemotherapy protocol } \\
\hline COSS $86(1988-1995)$ & 62 & 0.30883 & 57 & 0.62776 \\
\hline COSS 96 (1996-2006) & 73.5 & & 64 & \\
\hline \multicolumn{5}{|l|}{ Localization } \\
\hline Axial skeleton & 33.3 & & 22.2 & \\
\hline Upper extremity & 68.7 & 0.01626 & 56.3 & 0.0133 \\
\hline Lower extremity & 71.1 & & 65 & \\
\hline \multicolumn{5}{|l|}{ Gender } \\
\hline Boys & 66.2 & 0.68473 & 57 & 0.48097 \\
\hline Girls & 70.2 & & 65 & \\
\hline \multicolumn{5}{|l|}{ histological subtypes } \\
\hline Osteoblastic & 67.7 & & 64.5 & \\
\hline Chondroblastic & 62.5 & 0.96227 & 56.3 & 0.87902 \\
\hline Fibroblastic & 73.3 & & 60 & \\
\hline Else & 69 & & 58.6 & \\
\hline \multicolumn{5}{|c|}{ Response to pre-operative chemotherapy } \\
\hline Poor response & 55.3 & 0.00974 & 50 & 0.0182 \\
\hline Good response & 84 & & 77.3 & \\
\hline \multicolumn{5}{|l|}{ age at diagnosis } \\
\hline younger than 14 years & 77.6 & 0.04383 & 74.1 & 0.0085 \\
\hline older than 14 years & 59.4 & & 48.4 & \\
\hline
\end{tabular}




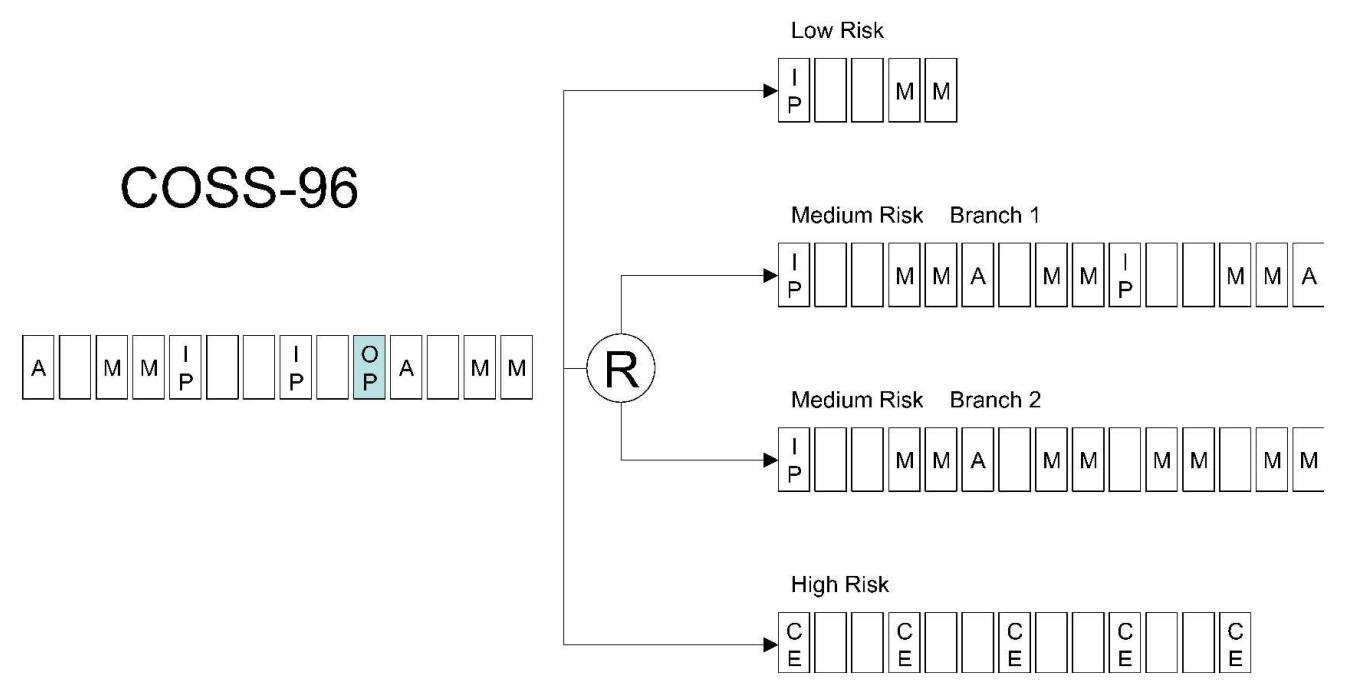

Figure 1. COSS 96 protocol.

A, doxorubicin $90 \mathrm{mg} / \mathrm{m} 2 ; C$, carboplatin $4 \times 150 \mathrm{mg} / \mathrm{m} 2 ; E$, etoposid $4 \times 150 \mathrm{mg} / \mathrm{m} 2 ;$ I, ifosfamide $2 \times 3 \mathrm{~g} / \mathrm{m} 2 ; \mathrm{M}$, high-dose methotrexate $12 \mathrm{~g} / \mathrm{m} 2$; OP: date of operation; P, cisplatin $120 \mathrm{mg} / \mathrm{m} 2$; R: randomization.

$359 \times 180 \mathrm{~mm}(600 \times 600 \mathrm{DPI})$ 


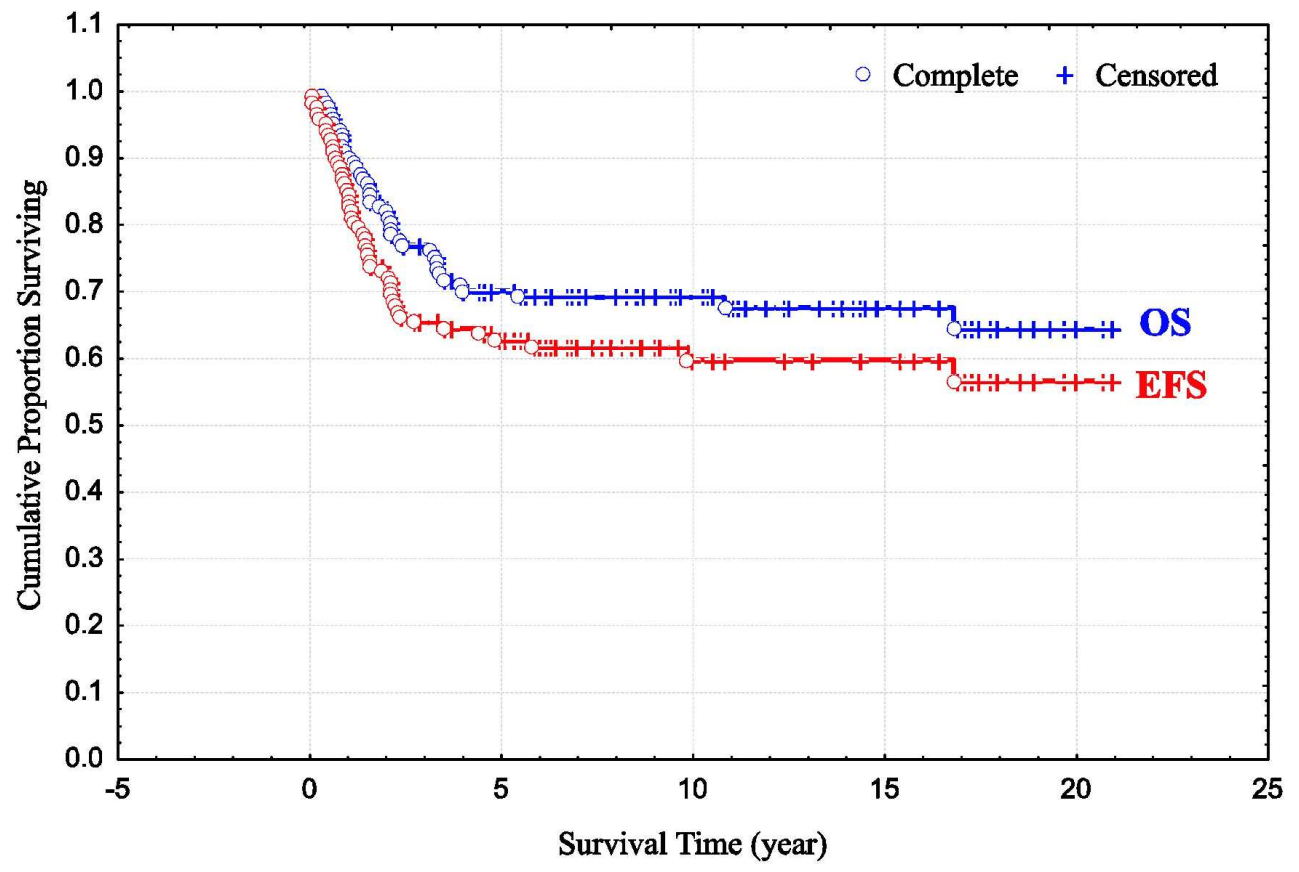

Figure 2. Survival of all patients with osteosarcoma. OS $=68 \%$ (blue line), EFS $=62 \%$ (red line). $151 \times 105 \mathrm{~mm}(600 \times 600 \mathrm{DPI})$ 
$x$

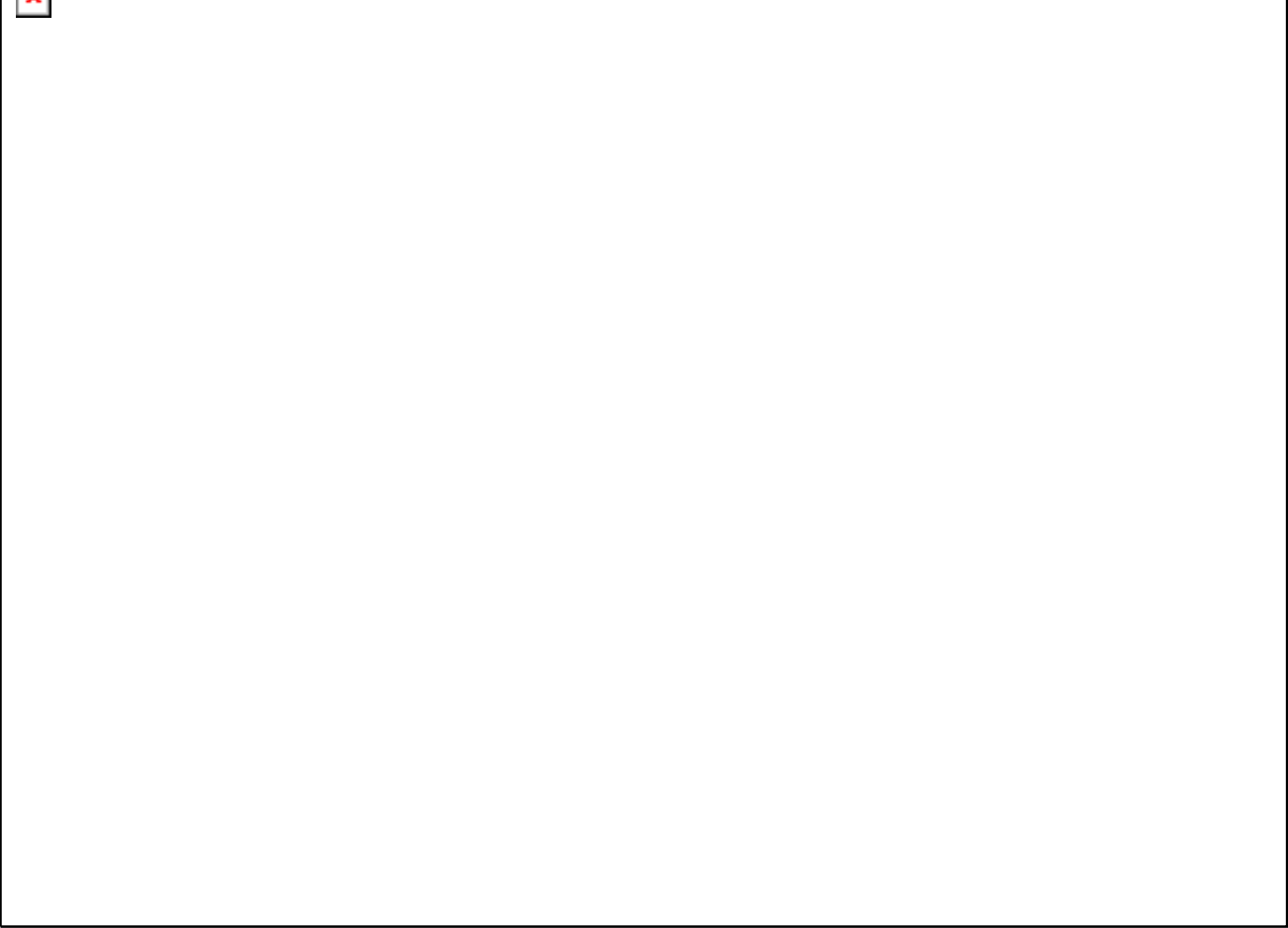

Figure 3. Flow diagram of patients with osteosarcoma treated at the Second Department of Pediatric in Budapest between 1988-2006. $160 \times 120 \mathrm{~mm}(600 \times 600 \mathrm{DPI})$ 

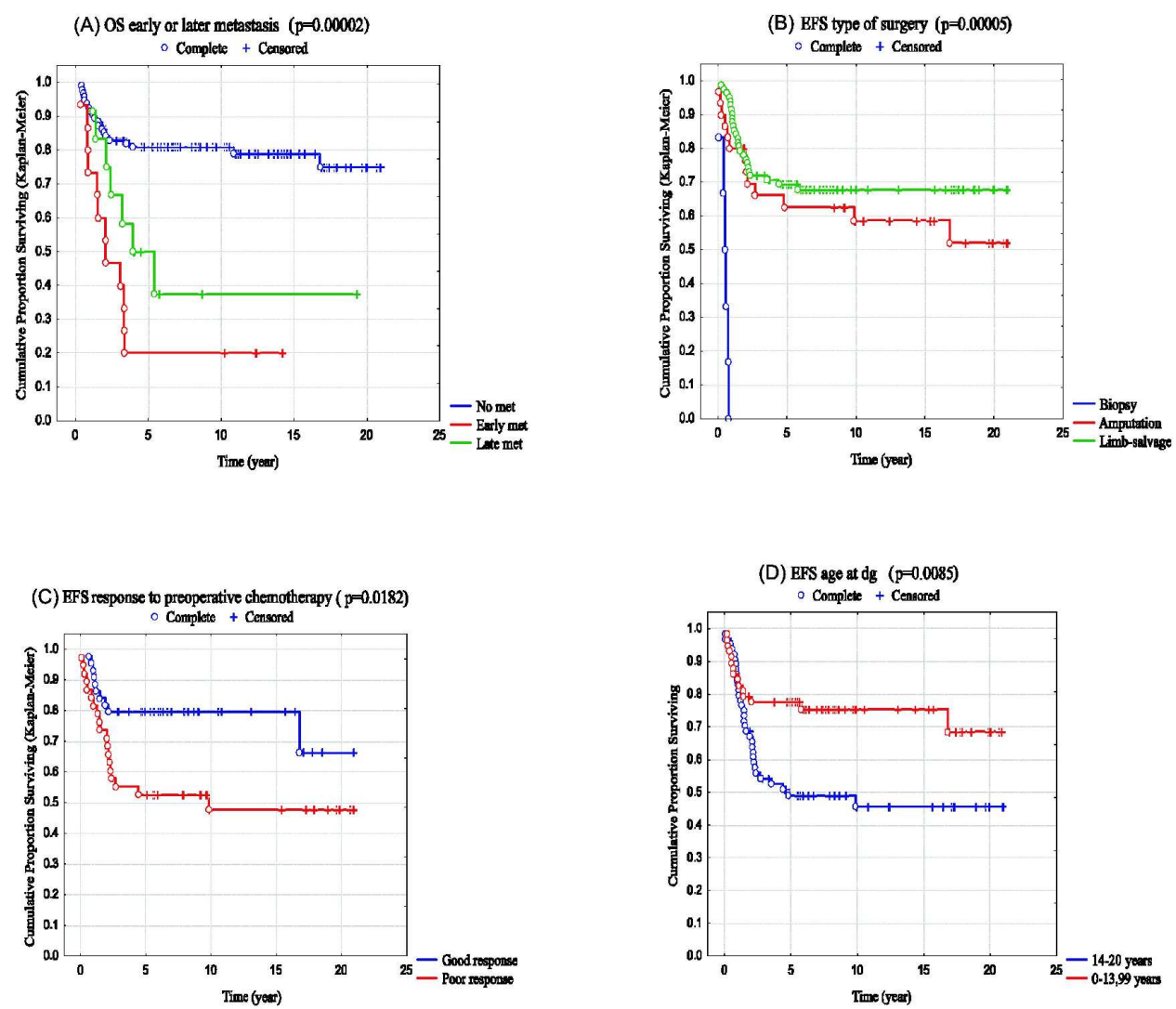

Figure 4. Survival of patients by factors with significant prognostic value.

(A) OS of patients by metastasis, $p=0.00002$. No met (blue line) OS $=78.95 \%$, early met (red line) OS $=16.66 \%$, late met (green line) OS $=41.66 \%$; (B) EFS of patients according to the type of surgery, $\mathrm{p}=0.00005$. Biopsy (blue line) $\mathrm{EFS}=0.00 \%$, amputation (red line) $\mathrm{EFS}=$ $56.66 \%$, limb-salvage surgery (green line) EFS $=68.29 \%$; (C) EFS by response to pre-operative chemotherapy, $p=0.0182$. Poor response (red line) EFS $=50.00 \%$, good response (blue line) EFS $=77.27$; (D) EFS by age at diagnosis, $p=0.0085$, younger than 14 years (red line) $\mathrm{EFS}=74.14 \%$, older than 14 years (blue line) EFS $=48.4 \%$; Abbreviations: EFS, event-free survival; met, metastasis; OS, overall survival. $152 \times 133 \mathrm{~mm}(600 \times 600 \mathrm{DPI})$ 


\section{$x$}

Figure 5. Survival of patients according to factors without significant prognostic value. (A) EFS by date of diagnosis $p=0.62776$, Dg between 1988-1995 (blue line) $\mathrm{EFS}=56.90 \%, \mathrm{Dg}$ between 1996-2006 (red line) EFS $=64.06 \%$; (B) EFS by gender $p=0.48097$. Girls (blue line) EFS $=64.91 \%$, boys (red line) EFS $=56.92 \%$; (C) EFS by histological subtype, $p=0.87902$, osteoblastic (blue line) $\mathrm{EFS}=64.56 \%$, chondroblastic (red line) $\mathrm{EFS}=56.25 \%$, fibroblastic (green line) $\mathrm{EFS}=60.00 \%$, other (purple line) $\mathrm{EFS}=58.62 \%$; (D) EFS by localization, $p=0.7$, upper extremity (red line) $\mathrm{EFS}=56.25 \%$, lower extremity (green line) $\mathrm{EFS}=64.94 \%$, axial skeleton (blue line) $\mathrm{EFS}=22.22 \%(p=0.0133)$; abbreviations: Dg, diagnosis; EFS, event-free survival. $158 \times 136 \mathrm{~mm}(600 \times 600 \mathrm{DPI})$ 